\section{PM0-022 ARE RECOMMENDED GUIDELINES FOR IV PARACETAMOL PRESCRIPTION FOLLOWED IN SUSCEPTIBLE GASTROENTEROLOGY PATIENTS?}

doi:10.1136/gutjnl-2012-302514b.22

${ }^{1} \mathrm{~J}$ L Burgess, ${ }^{*} \mathrm{~A}$ I Thuraisingam, ${ }^{1} \mathrm{P}$ Akhurst, ${ }^{1} \mathrm{M}$ Bunn. ${ }^{1}$ Department of Medicine, NHS, Wirral, UK; ${ }^{2}$ Department of Gastroenterology, NHS, Wirral, UK

Introduction IV Paracetamol use has increased since gaining EU drug approval in 2002, possibly due to its rapid onset and favourable sideeffect profile. ${ }^{1}$ The recommended adult daily dose is $4 \mathrm{~g} /$ day. The standard daily dose may be high enough to cause acute liver failure in patients with certain Gastroenterological problems [hepatocellular insufficiency, chronic malnutrition ${ }^{2}$ (The British National Formulary (BNF) version 60 advises that IV Paracetamol should be reduced to $3 \mathrm{~g} /$ day in these susceptible patients. ${ }^{3}$ We aimed to assess if IV paracetamol prescriptions are in line with BNF guidance.

Methods A prospective, point prevalence audit of all patients receiving IV paracetamol was undertaken on 2 days, 2 weeks apart. All adult inpatients (excluding the Women's Department) at Arrowe Park Hospital were assessed. A list of all patients receiving IV paracetamol was identified through the electronic prescribing system. The case-notes of all these patients were reviewed for evidence of high-risk characteristics including; weight.

Results Over two audit days a total of 104 out of 1158 (9\%) hospital inpatients were prescribed IV Paracetamol. Three patients were present on both days, therefore 101 sets of data were collected. 16 patients (16\%) prescribed IV Paracetamol were identified as high risk (hepatocellular insufficiency, chronic malnutrition or chronic alcoholism), and 15 (94\%) of these patients were not prescribed a reduced dose. This equates to a drug error in $1.3 \%$ of all inpatients. Conclusion IV Paracetamol is prescribed very frequently. Prescription is common in susceptible Gastroenterology patients with malnutrition, liver disease and alcoholism. Almost all susceptible patients in this audit were prescribed an inappropriately high dosage of paracetamol. This may lead to significant adverse side effects in an already vulnerable group of patients. It is important to increase awareness of reduced dosages in susceptible patients. Locally an alert has been placed on the electronic prescribing system and we have increased Pharmacist and medical staff awareness of appropriate paracetamol doses. We suspect our audit findings may be similar in other hospitals.

Competing interests None declared.

\section{REFERENCES}

1. Schmitt E, Vainchtock A, Nicoloyannis N, et al. Ready to use injectable paracetamol: easier, safer, lowering workload and costs. EJHP 2003;6:96-102.

2. Claridge LC, Eksteen B, Smith $A$, et al. Acute liver failure after administration of paracetamol at the maximum recommended daily dose in adults. BMJ 2010;341:1269-70.

3. BNF 60. London: BMJ Group and Pharmaceutical Press, 2010.

\section{PM0-023 ASSESSMENT OF QUALITY OF LIFE, COPING STRATEGIES AND PERSONAL BELIEFS IN NEUROENDOCRINE TUMOUR PATIENTS}

doi:10.1136/gutjnl-2012-302514b.23

J Garcia-Hernandez,* M Khan, J Goodhand, C Toumpanakis, M Caplin. Royal Free Hospital, London, UK

Introduction Medical research is focused on the developing of treatments and improving survival outcomes. In recent years, quality of life (QoL) measurements have been included in clinical trials as a relevant outcome variable; however, other psychological variables might have an impact on QoL. This study aims to determine: H1. Whether NET symptoms have a greater impact on QoL than disease severity. H2. Whether coping strategies influence QoL. H3 Whether internal control beliefs in the potential of control cancer growth are associated with QoL.

Methods 74 patients completed a web survey involving: QoL (EORTC C.30 and GI.NET21), a coping styles (Brief cope) and an illness perception measurements. All questionnaires were counterbalanced.

Results Endocrine and gastrointestinal symptoms, were negatively associated with self-reported measures of QoL, $r=-46, N=73$, p.05. Problem focus strategies such as acceptance, active coping and planning were not associated with QoL. Interestingly, emotion focus strategies such as behavioural disengagement, venting and self-blame were negatively associated with $\mathrm{OoL}, \mathrm{r}=-0.48, \mathrm{~N}=73$. Neuroendocrine patients considered that, keeping a positive attitude, not smoking and limiting alcohol intake would prevent cancer from spreading and progressing; however none of them were correlated with QoL.

Conclusion Disease severity is not associated with to QoL, whereas specific neuroendocrine symptoms, flushing and diarrhoea are. Active coping, acceptance, seeking emotional or instrumental support, were not correlated to QoL. In contrast, behavioural disengagement, denial and self-blame were negatively associated to self reported measures of QoL. Most of our patients endeavour on active or problem focus strategies where there is agreement of being more adaptive long term than avoidant or emotional strategies. This study has shown the relationship between neuroendocrine symptoms and coping strategies with QoL. Prospective and randomised trials will clarify the causal relationship among these constructs. Future research should include the relationship of psychosocial variables and neuroendocrine biomarkers.

Competing interests None declared.

\section{REFERENCES}

1. Carver CS. You want to measure coping but your protocol is too long: Consider the Brief COPE. Int J Behav Med 1997; 4:92-100

2. Larsson G, Sjoden PO, Oberg K, et al. Importance-satisfaction discrepancies are associated with health-related quality of life in five year survivors of endocrine gastrointestinal tumours. Ann Oncol 1999;10:1321-7.

3. Miles A, Simon A, Wardle J. Answering patient questions about the role lifestyle factors play in cancer onset and recurrence. J Health Psychol. 2010;15:291-8.

\section{PMO-024 A QUALITATIVE EXPLORATION OF INFLAMMATORY BOWEL DISEASE PATIENT ATTITUDES TOWARDS SELF MANAGEMENT AND NEW MODES OF CARE DELIVERY}

doi:10.1136/gutjnl-2012-302514b.24

${ }^{1} \mathrm{~K}$ Kemp, ${ }^{*}{ }^{2} \mathrm{~J}$ Griffiths, ${ }^{3} \mathrm{~S}$ Campbell, ${ }^{2} \mathrm{~K}$ Lovell. ${ }^{1}$ Department of Gastroenterology, School of Nursing, University of Manchester, Manchester Royal Infirm, Manchester UK; ${ }^{2}$ School of Nursing, University of Manchester, Manchester, UK; ${ }^{3}$ Gastroenterology, Manchester Royal Infirmary, Manchester, UK

Introduction Equity and excellence: Liberating the NHS (2010) states that new modes of care delivery must be explored for patients with long term conditions (LTCs). Increasing the proportion of people with a LTC to self care is a Quality Indicator and High Level Outcome in the Health and Well Being Strategy. The increasing focus on self care and reduction in inappropriate outpatient appointments are key priorities in commissioning services. The role of self management in IBD has been established but patient perceptions of self management programmes need to be addressed when implementing new modes of care delivery.

Methods The aim of this study was explore patient perceptions of self management as part of a follow-up care model. 24 in depth qualitative interviews were conducted, 18 patients had Crohn's Disease, six ulcerative colitis, age range $27-72$ yrs. Disease duration range was $2-40$ years. Patients were asked about self management, 